\title{
Cortical and Subcortical Effects of Transcutaneous Spinal Cord Stimulation in Humans with Tetraplegia
}

\author{
Francisco D. Benavides, ${ }^{1,2,3}$ Hang Jin Jo, ${ }^{1,2,3}$ Henrik Lundell, ${ }^{4}$ V. Reggie Edgerton, ${ }^{5,6,7,8,9,10}$ ○Yuri Gerasimenko, ${ }^{11,12,13}$ \\ and Monica A. Perez ${ }^{1,2,3}$ \\ ${ }^{1}$ Department of Neurological Surgery, The Miami Project to Cure Paralysis, University of Miami, Miami VA Medical Center, Miami, Florida 33136, 2Shirley \\ Ryan Ability Lab, Northwestern University, Chicago, Illinois 60611, ${ }^{3}$ Edward J. Hines, Jr. VA Medical Center, Chicago, Illinois 60141, ${ }^{4}$ Danish Research \\ Centre for Magnetic Resonance, Centre for Functional and Diagnostic Imaging and Research, Copenhagen University Hospital Hvidovre, 2650 Hvidovre, \\ Denmark, ${ }^{5}$ Department of Integrative Biology and Physiology, University of California, Los Angeles, Los Angeles, California 90095-7246, ${ }^{6}$ Department of \\ Neurobiology, University of California Los Angeles, Los Angeles, California 90095-1763, 7Department of Neurosurgery and ${ }^{8}$ Brain Research Institute, \\ University of California Los Angeles, Los Angeles, California 90095-1761, 9 Institut Guttmann, Hospital de Neurorehabilitació, Universitat Autònoma de \\ Barcelona, 08916 Badalona, Spain, ${ }^{10}$ Centre for Neuroscience and Regenerative Medicine, Faculty of Science, University of Technology Sydney, Sydney \\ 2007, Australia, and ${ }^{11}$ Pavlov Institute of Physiology, St. Petersburg 197022, Russia, ${ }^{12}$ Department of Physiology, University of Louisville, Louisville, \\ Kentucky 40292, and ${ }^{13}$ Kentucky Spinal Cord Injury Research Center, University of Louisville, Louisville, Kentucky 40292
}

An increasing number of studies supports the view that transcutaneous electrical stimulation of the spinal cord (TESS) promotes functional recovery in humans with spinal cord injury (SCI). However, the neural mechanisms contributing to these effects remain poorly understood. Here we examined motor-evoked potentials in arm muscles elicited by cortical and subcortical stimulation of corticospinal axons before and after 20 min of TESS ( $30 \mathrm{~Hz}$ pulses with a $5 \mathrm{kHz}$ carrier frequency) and sham-TESS applied between $\mathrm{C} 5$ and $\mathrm{C} 6$ spinous processes in males and females with and without chronic incomplete cervical SCI. The amplitude of subcortical, but not cortical, motorevoked potentials increased in proximal and distal arm muscles for $75 \mathrm{~min}$ after TESS, but not sham-TESS, in control subjects and SCI participants, suggesting a subcortical origin for these effects. Intracortical inhibition, elicited by paired stimuli, increased after TESS in both groups. When TESS was applied without the $5 \mathrm{kHz}$ carrier frequency both subcortical and cortical motor-evoked potentials were facilitated without changing intracortical inhibition, suggesting that the $5 \mathrm{kHz}$ carrier frequency contributed to the cortical inhibitory effects. Hand and arm function improved largely when TESS was used with, compared with without, the $5 \mathrm{kHz}$ carrier frequency. These novel observations demonstrate that TESS influences cortical and spinal networks, having an excitatory effect at the spinal level and an inhibitory effect at the cortical level. We hypothesized that these parallel effects contribute to further the recovery of limb function following SCI.

Key words: corticospinal; intracortical inhibition; neurophysiology; neuroplasticity; spinal cord injury; spinal networks

\section{Significance Statement}

Accumulating evidence supports the view that transcutaneous electrical stimulation of the spinal cord (TESS) promotes recovery of function in humans with spinal cord injury (SCI). Here, we show that a single session of TESS over the cervical spinal cord in individuals with incomplete chronic cervical SCI influenced in parallel the excitability cortical and spinal networks, having an excitatory effect at the spinal level and an inhibitory effect at the cortical level. Importantly, these parallel physiological effects had an impact on the magnitude of improvements in voluntary motor output.

\section{Introduction}

An increasing number of studies support the view that transcutaneous electrical stimulation of the spinal cord (TESS) promotes

Received Oct. 3, 2019; revised Jan. 9, 2020; accepted Jan. 17, 2020.

Author contributions: F.D.B., H.J.J., V.R.E., Y.G., and M.A.P. designed research; F.D.B., H.J.J., and M.A.P. per-

formed research; F.D.B., H.J.J., H.L., and M.A.P. analyzed data; F.D.B., H.J.J., and M.A.P. wrote the paper.

M.A.P. received funding from National Institute of Neurological Disorders and Stroke, the Veterans Administra- functional recovery in humans with spinal cord injury (SCI). TESS has shown improvements in sensory and motor function

\footnotetext{
tion, and the Walkabout Foundation. H.L. received funding from the European Research Council under the European Union's Horizon 2020 Research and Innovation Programme.

The authors declare no competing financial interests.

Correspondence should be addressed to Monica A. Perez at mperez04@sralab.org.

https://doi.org/10.1523/JNEUROSCI.2374-19.2020

Copyright $\odot 2020$ the authors
} 
when it is applied alone or in combination with other therapies (Hofstoetter et al., 2013; Gerasimenko et al., 2015; Gad et al., 2017, 2018; Inanici et al., 2018). Improved cardiovascular (Phillips et al., 2018) and bladder (Horst et al., 2013; Radziszewski, 2013) functions and reduced spasticity (Hofstoetter et al., 2014, 2020) have also been observed. While it is clear that single TESS pulses generate spinally mediated responses activating spinal networks that project transsynaptically to motoneurons (Milosevic et al., 2019), the effects of repeated use of TESS on CNS pathways remain unknown. A more complete understanding of the neural mechanisms of TESS-induced recovery is likely to enhance rehabilitation effectiveness.

Electrophysiological (Minassian et al., 2007a; Hofstoetter et al., 2018) and modeling (Rattay et al., 2000; Capogrosso et al., 2013) studies suggest that a single TESS pulse recruits large-tomedium-diameter proprioceptive and cutaneous afferents within posterior rootlets/roots of different spinal segments depending on the stimulus intensity. Most studies in humans with SCI aiming to achieve therapeutic effects use TESS currents between 5 and $50 \mathrm{~Hz}$ with intensities fluctuating between 10 and $200 \mathrm{~mA}$ using a high carrier frequency between 5 and $10 \mathrm{kHz}$ for several minutes to hours (see references above). In humans, a prolonged period of repeated stimulation of afferent fibers in a peripheral nerve with frequencies between 5 and $50 \mathrm{~Hz}$ usually increases the net excitability of descending cortical-motor neuron pathways, at least in part by reducing the activity of GABAergic cortical mechanisms (Kaelin-Lang et al., 2002; Golaszewski et al., 2012). Note that TESS uses a high carrier frequency or a "Russian current". Russian currents are alternating currents in a frequency range from $\sim 100 \mathrm{~Hz}$ to $10 \mathrm{kHz}$ that are delivered in bursts, with a burst frequency within a "physiological" range (up to $\sim 100 \mathrm{~Hz}$; Ward et al., 2009). It is thought that this high carrier frequency is beneficial for improving muscle strength (Selkowitz, 1985, 1989) and for suppressing the sensitivity of pain receptors (Ward and Robertson, 1998a,b). High-frequency electrical stimulation of the spinal cord releases serotonin in the dorsal horn (Linderoth et al., 1993), which may depress ascending nociceptive transmission (Fürst, 1999; Millan, 2002). Indeed, stimulation of muscle afferents at frequencies $\sim 100 \mathrm{~Hz}$ has an inhibitory effect on motor cortex (Mima et al., 2004). We hypothesized that TESS has a predominant excitatory effect on spinal networks and predominantly inhibitory effects at the cortical level, both of which could serve to fine-tune the net output that could contribute to further improving functional recovery.

To test our hypothesis, we evoked motor potentials in arm muscles by cortical and subcortical stimulation of corticospinal axons before and after 20 min of TESS $(30 \mathrm{~Hz}$ pulses with a $5 \mathrm{kHz}$ carrier frequency) applied between C5 and C6 spinous processes in humans with and without chronic incomplete cervical SCI. Structural magnetic resonance imaging (MRI) was used to localize the extent of the spinal cord lesion and to ensure that TESS was applied over injured segments.

\section{Materials and Methods}

Participants. Seventeen individuals with SCI participants (mean age, $43.1 \pm 14.0$ years; 4 females) and 15 age-matched control subjects (mean age, $36.5 \pm 17.5$ years; 6 females; $p=0.3$ ) participated in the study (Table 1). All participants gave informed consent to experimental procedures, which were approved by the local ethics committee at the University of Miami in accordance with guidelines established in the Declaration of Helsinki. Participants with SCI had a chronic injury ( $\geq 1$ year) and were classified using the International Standards for Neurological Classification of Spinal Cord Injury examination as having a C4-C6 SCI and by the American Spinal Cord Injury Association Impairment Scale (AIS) as AIS
Table 1. Spinal cord injury participants

\begin{tabular}{|c|c|c|c|c|c|c|c|c|c|c|}
\hline & & & & & & Postinjury & & & & \\
\hline Participant & (years) & Gender & AIS & Level & Etiology & (years) & Medication & M-max & MVC & MRI \\
\hline 1 & 39 & M & A & $C 5$ & $\mathrm{~T}$ & 12 & None & 30.8 & 1.1 & $X$ \\
\hline 2 & 60 & $M$ & D & $C 5$ & $\mathrm{~T}$ & 16 & $\mathrm{Bac}$ & 10.5 & 1.2 & $X$ \\
\hline 3 & 47 & M & B & C5 & $\mathrm{T}$ & 5 & $\mathrm{Bac}$ & 11.2 & 0.3 & $X$ \\
\hline 4 & 55 & $F$ & D & C5 & $\mathrm{T}$ & 17 & None & 10.0 & 0.6 & $X$ \\
\hline 5 & 42 & M & D & C5 & $\mathrm{T}$ & 18 & None & 23.0 & 0.9 & $X$ \\
\hline 6 & 32 & M & B & C5 & $\mathrm{T}$ & 19 & None & 9.0 & 0.8 & $X$ \\
\hline 7 & 45 & $M$ & $C$ & (4 & $\mathrm{T}$ & 11 & None & 15.2 & 0.8 & $X$ \\
\hline 8 & 34 & $\mathrm{~F}$ & B & (4 & $\mathrm{T}$ & 16 & None & 21.9 & 1.1 & $X$ \\
\hline 9 & 28 & $\mathrm{~F}$ & $C$ & C4 & $\mathrm{T}$ & 9 & $\mathrm{Bac}$ & 13.0 & 0.7 & $X$ \\
\hline 10 & 27 & M & A & C6 & $\mathrm{T}$ & 4 & $\mathrm{Bac}$ & 8.0 & 0.8 & $X$ \\
\hline 11 & 33 & M & A & C5 & $\mathrm{T}$ & 7 & None & 15.0 & 0.4 & $X$ \\
\hline 12 & 29 & M & A & C4 & $\mathrm{T}$ & 4 & None & 26.0 & 0.5 & $X$ \\
\hline 13 & 52 & $M$ & D & C4 & $\mathrm{T}$ & 16 & None & 14.0 & 0.9 & $X$ \\
\hline 14 & 64 & M & D & C6 & $\mathrm{T}$ & 11 & None & 9.0 & 0.4 & $X$ \\
\hline 15 & 62 & $M$ & D & C5 & $\mathrm{T}$ & 5 & GBP & 14.4 & 1.2 & $X$ \\
\hline 16 & 62 & $M$ & $D$ & $C 5$ & $\mathrm{~T}$ & 18 & None & 27.0 & 1.3 & $X$ \\
\hline 17 & 22 & $F$ & D & C4 & $\mathrm{T}$ & 6 & None & 16.0 & 0.4 & - \\
\hline
\end{tabular}

Bac, Baclofen; F, female; GBP, gabapentin; M, male; T, traumatic.

A $(n=4)$, AIS B $(n=3)$, AIS C $(n=2)$, or AIS D $(n=8)$. Five SCI individuals were taking antispastic medication (baclofen and/or gabapentin and/or tizanidine; Table 1).

Study design. All subjects participated in the main experiment, in which a $2 \mathrm{~d}$ crossover design was used to compare the effect of $20 \mathrm{~min}$ of TESS or sham-TESS, applied between C5 and C6 spinous processes, on motor-evoked potentials (MEPs) elicited by transcranial magnetic stimulation (TMS) and by cervicomedullary electrical stimulation (CMEPs) in the biceps brachii. Participants, but not experimenters, were blinded to the interventions. The duration of stimulation was comparable to that used in other plasticity protocols targeting the spinal cord in humans (Taylor and Martin, 2009; Bunday and Perez, 2012). The following tests were also completed in additional sessions in a subset of subjects: (1) we examined the effect of $20 \mathrm{~min}$ of TESS on MEPs and CMEPs elicited in the triceps brachii (control subjects $=10$; SCI participants $=11$ ) and first dorsal interosseous (control subjects $=8$; SCI participants $=8$ ) muscles This was tested separately because different coil locations and stimulus intensities are needed to elicit MEPs and CMEPs in the triceps brachii and the first dorsal interosseous than in the biceps brachii; (2) because MEP amplitude increased after TESS, but not sham-TESS, we examined the effect of $20 \mathrm{~min}$ of TESS on short-interval intracortical inhibition (SICI; control subjects $=14$; SCI participants $=17)$ in a separate session to assess the contribution of intracortical pathways to the observed changes in MEP amplitude; and (3) to assess the effect of TESS without the $5 \mathrm{kHz}$ carrier frequency (TESS without $5 \mathrm{kHz}$ ), we examined the effects of $20 \mathrm{~min}$ of TESS without $5 \mathrm{kHz}$ on MEPs and CMEPs and on SICI in separate sessions (control subjects $=8$; SCI participants $=8$ ). Subjects tested on these sessions were randomly selected.

Electromyographic (EMG) recordings. Electromyograms were recorded from the biceps brachii muscle of the right side in control subjects and from the less affected hand in individuals with SCI through surface electrodes secured to the skin over the belly of each muscle (Ag-AgCl, 10 $\mathrm{mm}$ diameter). To examine whether the effects of TESS spread to other muscles, we also recorded from the triceps brachii and the first dorsal interosseous. The signals were amplified, filtered $(30-2000 \mathrm{~Hz})$, and sampled at $10 \mathrm{kHz}$ for offline analysis (CED 1401 with Signal software, Cambridge Electronic Design). Subjects performed three brief elbow flexion maximal voluntary isometric contractions (MVCs) for 3-5 s, separated by $\sim 30$ s of rest. The maximal mean EMG activity of the rectified response generated for $1 \mathrm{~s}$ during each MVC was analyzed, and the highest value of the three trials was used. The MVC with the biceps brachii during elbow flexion was larger in control subjects $(1.4 \pm 0.5$ $\mathrm{mV})$ compared with SCI participants $(0.8 \pm 0.4 \mathrm{mV} ; p=0.007)$.

Experimental setup. Subjects were seated comfortably in an armchair with both arms placed on a custom platform with the elbow and shoulder flexed at $90^{\circ}$ (Fig. $1 A$ ). Individuals randomly received TESS and sham- 
A

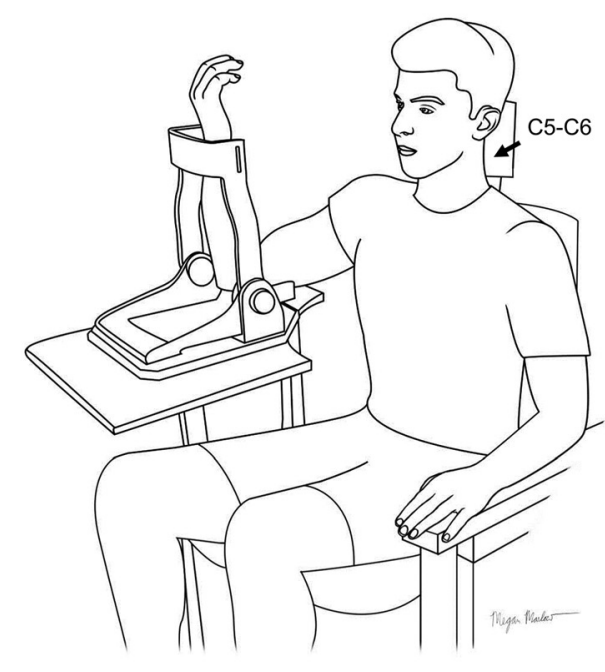

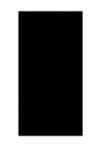

Pre
B

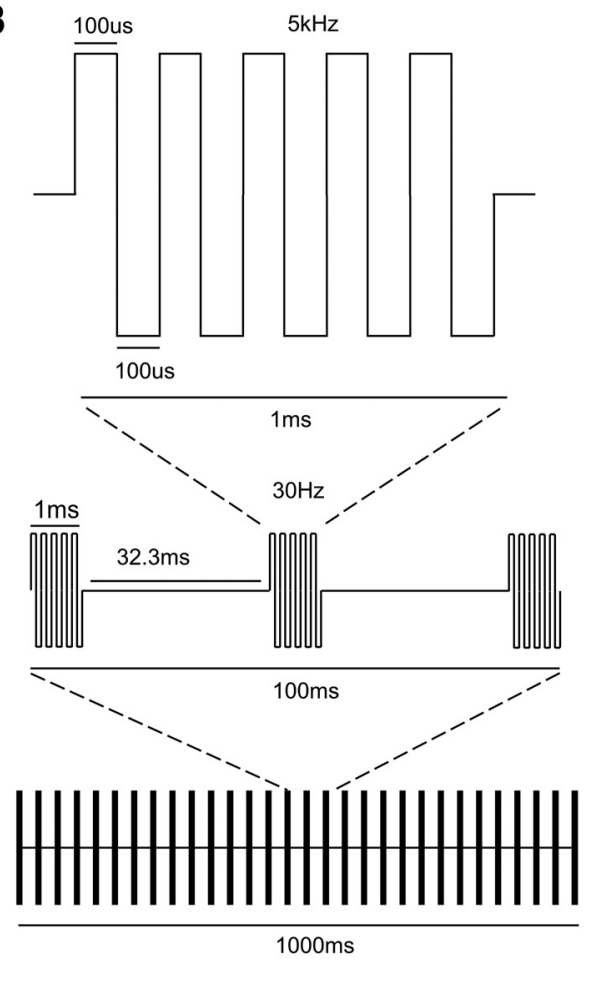

Figure 1. Experimental setup. A, Participants were comfortably seated in a customized chair during TESS or sham-TESS for $20 \mathrm{~min}$. TESS was delivered using a surface electrode on the back of the neck between $C 5$ and $(6$ spinous processes segments (cathode) and a surface electrode in each anterior crest of the hip bone (anode) using a custom-made five-channel stimulator (BioStim5). Electrophysiological and behavioral outcomes were tested Pre, immediately after, and 15, 30, 45, 60, and 75 min after the end of the stimulation or sham stimulation period. B, Schematic representation of the type of current used during TESS. We used five biphasic pulses at $5 \mathrm{kHz}$ with each biphasic pulse lasting for $200 \mu \mathrm{s}$. The middle scheme shows the blocks of five biphasic pulses passed at a $30 \mathrm{~Hz}$ frequency. The bottom part of the schematic shows the number of pulses delivered in $1 \mathrm{~s}$.

TESS on different sessions separated by $2-3 \mathrm{~d}$. During the TESS session, a custom-made five-channel constant current stimulator (BioStim-5, BioMedical Life Systems; Grishin et al., 2017) was used to noninvasively stimulate cervical spinal networks. Stimulation was delivered between the spinous process of $\mathrm{C} 5-\mathrm{C} 6$ using a 3.2-cm-diameter hydrogel adhesive electrode as cathode and two $7.5 \times 12 \mathrm{~cm}$ rectangular electrodes (ValuTrode Cloth, Axelgaard Manufacturing) located bilaterally over the iliac crests as anodes. We used blocks of five biphasic pulses (each pulse of 200 $\mu$ s duration) at a frequency of $30 \mathrm{~Hz}$ (every $32.3 \mathrm{~ms}$ ) for $20 \mathrm{~min}$ (Fig. 1B). Thus, each block was filled with a $5 \mathrm{kHz}$ carrier frequency. The stimulus intensity from a single biphasic pulse was defined as the minimal intensity required to induce a root-evoked potential in the biceps brachii $\geq 50$ $\mu \mathrm{V}$ peak-to-peak amplitude above the background EMG in 5 of 10 consecutive trials in the relaxed muscle $[$ control subjects $=77.5 \pm 10.8 \mathrm{~mA}$ (range, 55-90 mA); SCI participants $=79.2 \pm 12.2 \mathrm{~mA}$ (range, 55-95 $\mathrm{mA}) ; p=0.6]$. During the sham-TESS session, the electrodes were located in the same position, and the intensity of stimulation was set as the same intensity as used in TESS sessions and gradually decreased down to 0 in $\sim 1$ min. Similar sham stimulation procedures have been used successfully in previous neuromodulation studies using TESS to provide the initial sensation of stimulation without the subsequent effects (Murray et al., 2018; Awosika et al., 2019). During TESS without $5 \mathrm{kHz}$ carrier frequency (tested on subjects listed as 1-8 on Table 1), single biphasic pulses (200 $\mu$ s duration) were delivered at a $30 \mathrm{~Hz}$ frequency (every $33.1 \mathrm{~ms}$ ) for 20 min using the same intensity as described above. This paradigm was tolerated by all subjects, allowing us to match the same stimulation intensity as used in the original TESS protocol. By comparing the effects of TESS with and without the $5 \mathrm{kHz}$ carrier frequency, we can examine whether the additional pulses provided with the carrier frequency are causing any effects of physiological outcomes. Since changes in pulse duration can affect the relative recruitment of sensory axons, we maintained the waveform pulse duration consistent across paradigms (Paillard, 1955; Panizza et al., 1989). The following measurements were taken at rest before, immediately after, and up to $75 \mathrm{~min}$ after the end of each stimulation period: MEPs, CMEPs, SICI, and functional outcomes.

MEPs. Transcranial magnetic stimuli were delivered from a Magstim 200 Stimulator (Magstim Company) through a figure-of-eight coil (custom-made loop diameter, $7 \mathrm{~cm}$; type number SP15560) with a monophasic current waveform. TMS was delivered to the optimal scalp position for activation of the biceps brachii, triceps brachii, and first dorsal interosseous muscles as needed. The coil was held tangentially to the scalp to induce currents in the brain flowing in a posterior-anterior direction and held to the head of the subject with coil holder (Manfrotto) with the head firmly secured to a headrest to limit head movements. TMS stimuli were delivered at an intensity to generate MEPs of $\sim 3-5 \%$ of the maximal motor response (M-max) in the biceps brachii (control subjects $=4.4 \pm 1.5 \%$ of the M-max; SCI participants $=5.0 \pm 1.1 \%$ of the M-max; $p=0.9$ ), triceps brachii (control subjects $=2.9 \pm 1.9 \%$ of the M-max; SCI participants $=3.1 \pm 2.8 \%$ of the M-max; $p=0.4$ ), and the first dorsal interosseous (control subjects $=4.5 \% \pm 0.2$ of the $\mathrm{M}$ $\max$; SCI participants $=3.6 \pm 1.3 \%$ of the M-max; $p=0.1$ ) muscles. Peripheral nerve stimulation was delivered to the brachial plexus at the supraclavicular fossa to test the M-max in the biceps brachii (200 $\mu \mathrm{s}$ pulse duration; model DS7AH, Digitimer). The anode was placed at the Erb's point, and the cathode was placed over the acromion for biceps and triceps brachii M-max, and at the ulnar nerve at the wrist for the first dorsal interosseous (biceps brachii: control subjects $=21.7 \pm 4.7 \mathrm{mV}$; SCI participants $=18.2 \pm 4.8 \mathrm{mV} ; p=0.02$; triceps brachii: control subjects $=14.7 \pm$ $3.5 \mathrm{mV}$; SCI participants $=11.8 \pm 3.6 \mathrm{mV} ; p=0.03$; first dorsal interosseous: control subjects $=22.7 \pm 1.1 \mathrm{mV}$; SCI participants $=13.7 \pm 4.3 \mathrm{mV}$; $p<0.001)$. TMS pulses were delivered at $4 \mathrm{~s}$ intervals $(0.25 \mathrm{~Hz})$. Twenty MEPs were averaged at each time point before and after each protocol, and peak-to-peak MEP amplitude was measured.

CMEPs. CMEPs were elicited by simulation after elicited at the cervicomedullary junction by using high-voltage electrical current $(100 \mu \mathrm{s}$ duration; DS7AH, Digitimer) passed between adhesive $\mathrm{Ag}-\mathrm{AgCl}$ elec- 
trodes fixed to the skin behind the mastoid process. The stimulation intensity was set at an intensity to generate CMEPs of $\sim 3-5 \%$ of the $\mathrm{M}$-max in the biceps brachii (control subjects $=4.6 \pm 1.3 \%$ of the M-max; SCI participants $=5.2 \pm 1.0 \%$ of the M-max; $p=0.1$ ), triceps brachii $($ control subjects $=3.5 \pm 2.6 \%$ of the M-max; SCI participants $=$ $4.4 \pm 2.7 \%$ of the M-max; $p=0.2$ ), and the first dorsal interosseous (control subjects $=4.5 \pm 0.2 \%$ of the M-max; SCI participants $=3.6 \pm$ $1.3 \%$ of the M-max; $p=0.1$ ) muscles. We monitored the stimulation to ensure that it was below the intensity required to activate the peripheral nerve directly by increasing the intensity and observing a decrease in latency. The latency of CMEPs was shorter than the MEPs elicited by TMS in both groups in all muscles tested (biceps brachii, control subjects: MEPs $=13.0 \pm 0.8 \mathrm{~ms}$; CMEPs $=8.4 \pm 0.5 \mathrm{~ms} ; p<0.001$; SCI participants: $\mathrm{MEPs}=13.7 \pm 0.8 \mathrm{~ms}$; $\mathrm{CMEPs}=8.5 \pm 1.1 \mathrm{~ms} ; p<0.001$; triceps brachii, control subjects: $\mathrm{MEPs}=13.4 \pm 0.9 \mathrm{~ms}$; $\mathrm{CMEPs}=8.6 \pm 0.4 \mathrm{~ms}$; $p<0.001$; SCI participants: $\mathrm{MEPs}=14.4 \pm 1.1 \mathrm{~ms}$; CMEPs $=8.7 \pm 1.1$ $\mathrm{ms} ; p<0.001$; first dorsal interosseous, control subjects: $\mathrm{MEPs}=22.2 \pm$ $0.3 \mathrm{~ms}$; $\mathrm{CMEPs}=17.7 \pm 0.9 \mathrm{~ms} ; p<0.001$; SCI participants: MEPs $=$ $25.5 \pm 2.7 \mathrm{~ms}$; CMEPs $=20.0 \pm 1.8 \mathrm{~ms} ; p<0.001$ ). Five to $10 \mathrm{CMEPs}$ were averaged at each time point before and after each protocol for each muscle on separate sessions, and peak-to-peak CMEP amplitude was measured.

SICI. We tested SICI in biceps brachii using a previously described method (Kujirai et al., 1993). A conditioning stimulus (CS) was set at an intensity needed to elicit $\sim 50 \%$ of SICI, which corresponded to $\sim 80 \%$ of resting motor threshold. The same stimulation intensity was used before and after the TESS protocol. The test stimuli (TS) was set at an intensity needed to elicit an MEP that equals $\sim 3-5 \%$ of the M-max (control subjects $=4.6 \pm 1.1 \%$ of the M-max; SCI participants $=4.8 \pm 1.4 \%$ of the M-max; $p=0.3$ ). The delay between CS and TS was $2.5 \mathrm{~ms}$. SICI was calculated by expressing the amplitude of conditioned MEP as a percentage of the test MEP amplitude $[\%=($ conditioned MEP $\times 100) /($ test MEP)]. Two sets of 15 test MEPs and 15 conditioned MEPs were tested at each time point.

Functional outcomes. In the SCI group, we tested gross and fine motor functions (jar opening and water bottle test, and key test and coin test, respectively) using subcomponents of the Graded and Redefined Assessment of Strength, Sensibility and Prehension (GRASSP) test. During jar opening, subjects were asked to open a jar lid with a tested hand while holding the jar $(7 \mathrm{~cm}$ in diameter and $9 \mathrm{~cm}$ in height) with the nontested hand in the shortest time possible. For the water bottle test, subjects were asked to lift a $6-\mathrm{cm}$-diameter bottle, filled with $\sim 200 \mathrm{ml}$ of water, from the table and pour the water into a cup. During the key test, subjects were asked to pick up a key from the table, insert it in a lock, and give a full turn in the key slot. The coin test consisted in subjects picking up four coins, one by one, and positioning them into a slot as fast as possible. The order of three subcomponents of test was randomized in each subject and across subjects. Measurements were taken $30 \mathrm{~min}$ after the end of each session. The distance and position between each subject's hand and the apparatus was recorded and maintained constant for preassessments and postassessments.

MRI. Structural MRI was used to verify in SCI participants damage to C5-C6 spinal segments $(n=16)$. The images were acquired on a TIM Trio 3 T system using a 20-channel birdcage head/neck coil (Siemens). A T2-weighted turbo spin-echo sequence $(\mathrm{TR}=3 \mathrm{~s}$ echo time, $\mathrm{TE}=96$ $\mathrm{ms}$ ), with sagittal in-plane resolutions of 0.6 and $3 \mathrm{~mm}$ slice thickness, was used to evaluate spinal cord sections. Sagittal sections were used to determine the extension of the lesions in reference to the vertebral bodies, and each segment was limited by the position of the intervertebral discs and confirmed by two observers. The caudal extension of the injury was marked along the extension of each vertebra taking as reference superior and inferior borders. The position of C5-C6 was marked, and the distance from the center of the cord at this location to the surface of the skin as well as the distance to the subcutaneous (SC) fat was measured (Fig. 2A). Note that in SCI participants TESS was applied over injured spinal segments (Fig. 2B). One participant was unable to obtain the spinal cord MRI due to claustrophobia. Note that on this participant with a C4 neurological level of injury (participant 17 in Table 1) TESS electrodes were located between C5 and C6 spinous processes.

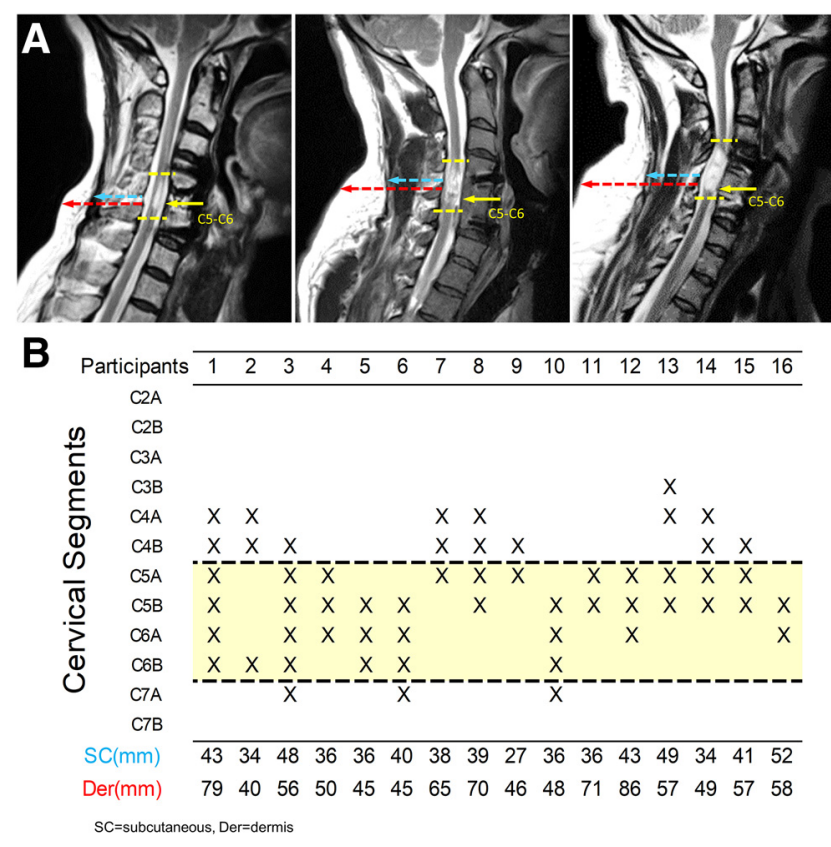

Figure 2. Structural MRI. $\boldsymbol{A}$, Three sagittal slices from representative participants with cervical SCl. The blue arrows show the distance from the canal toward the beginning of the SC space. The red arrows show the distance from the spinal canal toward the surface of the skin, including the cutaneous layer and fat tissue underneath. The yellow dotted lines show the top and bottom borders of the injury, and arrows point to the C5-C6 intervertebral space. Individual distances and segmental distributions of the injuries are shown in $\boldsymbol{B}$.

Data analysis. Normal distribution was tested by the Shapiro-Wilk test and homogeneity of variances by the Mauchly's test of sphericity. When sphericity could not be assumed, the Greenhouse-Geisser correction statistic was used. Repeated-measures ANOVA was performed to determine the effect of the group (control subjects and SCI participants), time [before stimulation (Pre), and at 15, 30, 45, 60, and 75 min after stimulation], and intervention (TESS and sham-TESS) on the amplitude of MEPs, CMEPs, and mean rectified background EMG and the effect of group and time on SICI. Repeated-measures ANOVA was also performed to determine the effect of the group, time, and intervention frequency (TESS, sham-TESS, and TESS without $5 \mathrm{kHz}$ ) on MEPs, CMEPs, SICI, and mean rectified background EMG; and the effect of intervention frequency and task (coin, key, and bottle) on the time to task completion. Note that statistical analysis was completed using the raw data, and when the data were normalized to the baseline (using 100\% values before the intervention) on each variable. Because the same results were found in both analyses, in the text we present the results using the raw data and in the figures we showed the data normalized to the baseline for clarity. Bonferroni post hoc tests were used to test for significant comparisons. Independent $t$ tests were used to compare age, M-max, MVC, and stimulation intensities (TMS and electrical stimulation). $t$ Test-corrected values were used when equal variances were not assumed. Pearson correlation analysis was used as needed. Significance was set at $p<0.05$. Group data are presented as the mean \pm SD in the text.

\section{Results}

MRI

Figure $2 A$ shows sagittal slices obtained from the cervical level of three representative participants with SCI. Measurements were taken to measure the depth from the surface of the skin to the spinal canal. Blue arrows show the distance from the canal toward the beginning of the SC space. This part is composed mainly of bone forming the laminae and the spinous process (isointense T2-weighted signal) as well as paravertebral muscles (hypointense T2-weighted signal). Red arrows show the distance from the spinal canal toward the surface of the skin (D) and included the 
A
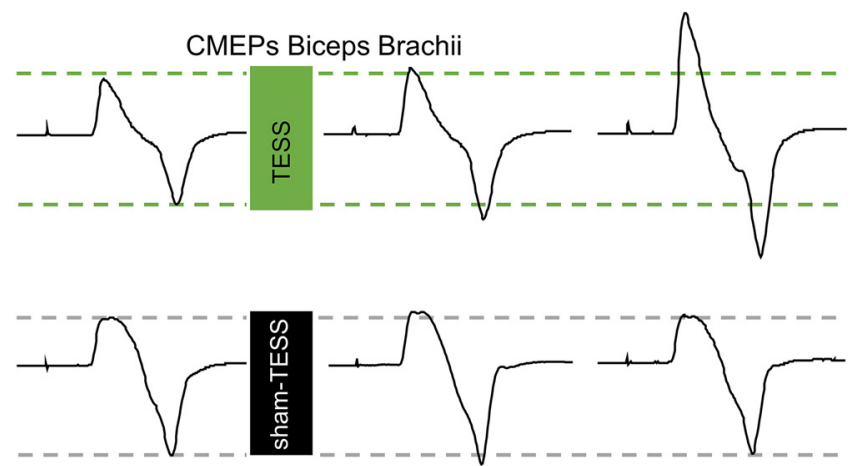

Pre
Control

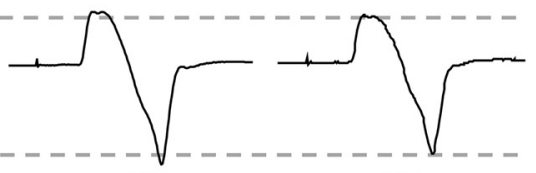

75

Time (min)
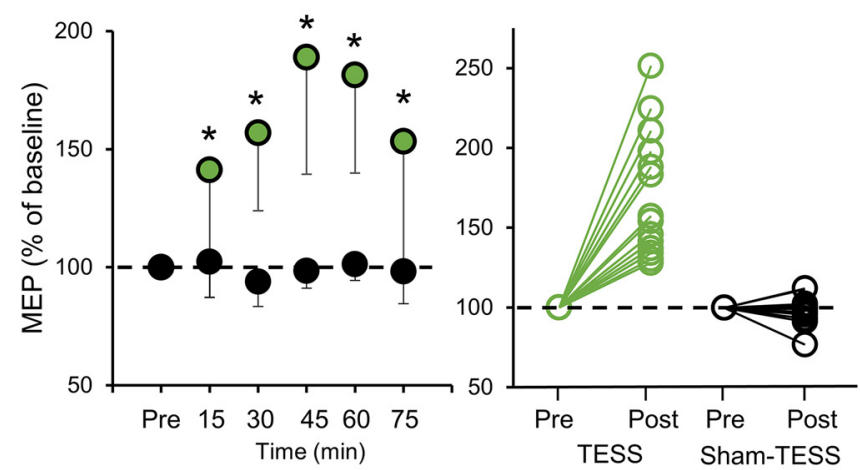

B

\section{Spinal Cord Injury}
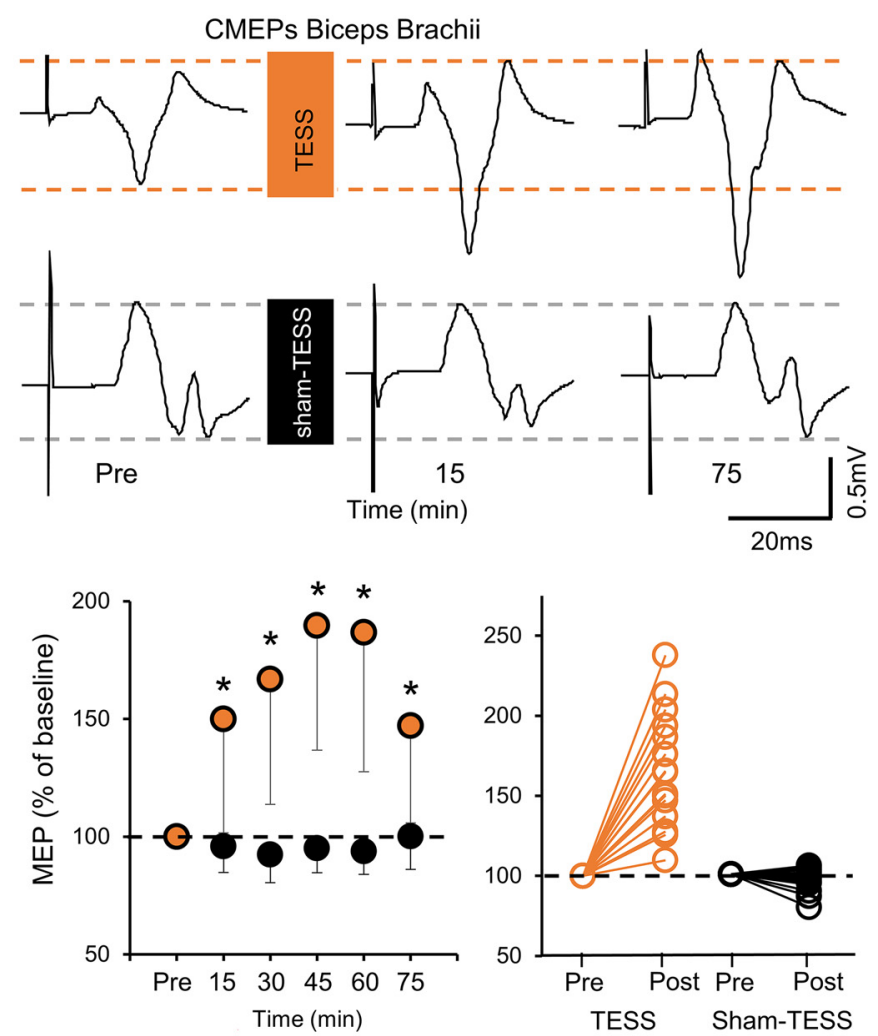

Figure 3. CMEPs. $\boldsymbol{A}, \boldsymbol{B}$, Averaged CMEP traces in the biceps brachii muscle in a representative control subject $(\boldsymbol{A})$ and a participant with SCI ( $\boldsymbol{B})$ before and after TESS and sham-TESS. Each waveform represents the average of $10 \mathrm{CMEPs}$. Graphs show group (control subjects, $n=15 ; \mathrm{SCl}, n=17$ ) and individual data. The ordinate shows the CMEP amplitude as a percentage of the CMEP at baseline (percentage of baseline) in control subjects and SCI participants before and after TESS (control subjects, green circles; SCI participants, orange circles) and sham-TESS (control subjects and SCI participants, black circles). The abscissa shows the time of measurements (Pre, and 15, 30, 45, 60, and 75 min after each protocol). Note that in graphs showing data from individual participant postmeasurement data showed the average from 15 to 75 min. Error bars indicate SDs. ${ }^{*} p<0.05$.

cutaneous layer and fat tissue underneath (hyperintense T2weighted signal). The yellow dotted lines show that the top and bottom borders of the injury and the arrow points to the C5-C6 intervertebral space. The distance from the spinal cord to the surface of the skin was variable from participant to participant, the thickness of the fat layer was calculated by subtracting D - SC $(\mathrm{SC}=39.5 \pm 6.4 \mathrm{~mm} ; \mathrm{D}=57.6 \pm 13.2 \mathrm{~mm}$; fat $=18.3 \pm 12.4$ $\mathrm{mm})$. We found a moderate but not significant correlation between SC and the amount of current needed to observe a threshold root-evoked potential in the biceps brachii muscle $(r=0.42$, $p=0.1)$. No correlations were found between $\mathrm{D}(r=0.01 p=$ $0.1)$ or fat $(r=0.06 p=0.8)$ and the amount of current needed to observe a threshold root-evoked potential in the biceps brachii muscle. Note that 13 participants showed injuries that extended more than one cervical segment, and all injuries were localized between $\mathrm{C} 4$ and $\mathrm{C} 7$ spinal segments (Fig. 2B).

\section{CMEPs}

Repeated-measures ANOVA showed an effect of intervention $\left(F_{(1,29)}=91.5, p<0.001\right)$ and time $\left(F_{(5,145)}=20.1, p<0.001\right)$, but not of group $\left(F_{(1,29)}=2.4, p=0.13\right)$ or in their interaction $\left(F_{(5,145)}=0.9, p=0.5\right)$ on biceps brachii CMEP amplitude. Additionally, there was an effect of intervention $\times$ time $\left(F_{(5,145)}=\right.$ 21.9, $p<0.001)$ without any other interactions. Our analysis revealed that CMEP amplitude increased after TESS in both groups at $15 \mathrm{~min}$ (control subjects $=141.2 \pm 54.0 \%, p<0.05$;
SCI participants $=150.0 \pm 48.4 \%, p<0.01$ ), $30 \mathrm{~min}$ (control subjects $=156.9 \pm 33.0 \%, p<0.001$; SCI participants $=166.9 \pm$ $53.2 \%, p<0.001), 45 \mathrm{~min}$ (control subjects $=188.9 \pm 49.4 \%$, $p<0.001$; SCI participants $=189.6 \pm 52.9 \% ; p<0.001), 60 \mathrm{~min}$ (control subjects $=181.4 \pm 41.4 \%, p<0.001$; SCI participants $=$ $186.7 \pm 59.2 \%, p<0.001$ ), and $75 \mathrm{~min}$ (control subjects $=$ $153.2 \pm 53.6 \%, p<0.001$; SCI participants $=147.1 \pm 41.2 \%$, $p<0.05)$ compared with baseline. In contrast, CMEP amplitude remained unchanged after sham TESS in both groups at $15 \mathrm{~min}$ $(98.7 \pm 13.5 \% ; p=0.4), 30 \mathrm{~min}(93.3 \pm 11.3 \% ; p=0.1), 45 \mathrm{~min}$ $(96.5 \pm 9.3 \% ; p=0.1), 60 \min (97.5 \pm 9.4 \% ; p=0.2)$, and 75 $\min (100.0 \pm 13.3 \% ; p=0.9)$ compared with baseline. Note that CMEPs were facilitated after TESS but not sham-TESS in all control and SCI participants, and representative examples and individual data are shown on Figure 3, $A$ and $B$ (top panels and lower graphs).

We observed similar increases in CMEP amplitude after TESS in triceps brachii [effect of time $\left(F_{(1.6,31.7)}=4.9, p<0.01\right)$ not Group $\left(F_{(1,20)}=1.9, p=0.2\right)$ or in their interaction $\left(F_{(1.6,31.7)}=\right.$ $1.1, p=0.3)$ ] and first dorsal interosseous [effect of time $\left(F_{(5,70)}\right.$ $=6.1, p<0.001)$ not group $\left(F_{(1,14)}=4.1, p=0.1\right)$ or in their interaction $\left.\left(F_{(5,70)}=1.3, p=0.3\right)\right]$ muscles. The amplitude of CMEPs increased on average in the triceps brachii in control subjects (166.6 $\pm 46.5 \%$; range, $100.6-243.6 \%)$ and SCI participants (150.9 \pm 43.3\%; range, 104.8-255.2\%) compared with baseline for up to $75 \mathrm{~min}$. Similarly, CMEPs in the first dorsal 
interosseous muscle were facilitated on average in control subjects (162.1 \pm 47.8\%; range, $113.3-243.9 \%)$ and SCI participants (130.6 $\pm 22.7 \%$; range, 101.3-160.2\%) compared with baseline measurements for up to $75 \mathrm{~min}$. Note that CMEP amplitude in all muscles was similar at baseline in both groups (biceps brachii: control subjects $=4.6 \pm 1.3 \%$ of the M-max; SCI participants $=5.2 \pm$ $1.0 \%$ of the M-max; $p=0.1$; triceps brachii: control subjects $=3.5 \pm 2.6 \%$ of the M-max; SCI participants $=4.4 \pm$ $2.8 \%$ of the M-max; $p=0.2$; first dorsal interosseous: control subjects $=5.4 \pm$ $0.8 \%$ of the M-max; SCI $=5.9 \pm 0.7 \%$ of M-max; $p=0.1$ ).

\section{MEPs}

Repeated-measures ANOVA revealed no effect of intervention $\left(F_{(1,30)}=0.1, p=\right.$ $0.8)$, time $\left(F_{(5,150)}=1.4, p=0.2\right)$, group $\left(F_{(1,30)}=0.7, p=0.4\right)$, or in their interaction $\left(F_{(5,150)}=0.3, p=0.9\right)$ on biceps brachii MEP amplitude. We observed no changes in MEP amplitude after TESS or sham-TESS up to $75 \mathrm{~min}$ in both groups tested (Fig. $4 A, B$ ). Similarly, we observed no effect of time $\left(F_{(1.5,30.6)}=1.0, p=0.4\right)$, group $\left(F_{(1,20)}=0.1\right.$, $p=0.7)$, or in their interaction $\left(F_{(1.5,30.6)}=1.9, p=0.2\right)$ on triceps brachii MEP amplitude. In addition, there was an effect of group $\left(F_{(1,12)}=11.3, p<0.01\right)$ but not of time $\left(F_{(5,60)}=1.2, p=\right.$ $0.3)$ or in their interaction $\left(F_{(5,60)}=0.4, p=0.8\right)$ on first dorsal interosseous MEP amplitude.

\section{SICI}

Repeated-measures ANOVA showed an effect of time $\left(F_{(5,145)}=\right.$ $15.5, p<0.001)$ and group $\left(F_{(1,29)}=10.8, p<0.01\right)$ but not in their interaction $\left(F_{(5,145)}=1.1, p=0.4\right)$ on SICI. We found that SICI increased after $15 \mathrm{~min}$ (control subjects $=55.9 \pm 30.9 \%$, $p<0.001$; SCI participants $=54.6 \pm 39.1 \%, p=0.02$ ), $30 \mathrm{~min}$ (control subjects $=44.9 \pm 37.8 \%, p<0.001$; SCI participants $=$ $54.0 \pm 40.0 \%, p<0.01$ ), $45 \mathrm{~min}$ (control subjects $=44.3 \pm$ $34.1 \%, p<0.001$; SCI participants $=53.7 \pm 24.0 \%, p<0.05), 60$ min (control subjects: $44.0 \pm 25.1 \%, p<0.001$; SCI participants $=61.7 \pm 39.0 \%, p<0.05)$, and $75 \mathrm{~min}$ (control subjects $=$ $55.5 \pm 25.3 \%, p<0.001$; SCI participants $=58.6 \pm 33.4 \%, p<$ $0.05)$ after TESS compared with baseline in control and SCI participants. Note that SICI was increased in all of subjects after the TESS protocol in the control group (14 of 14) and in most cases in SCI participants (13 of 15) when comparing baseline measurements with the average of measurements between 15 and 75 min after TESS (Fig. $5 A, B$, graphs).

\section{Effects without the carrier frequency}

Repeated-measures ANOVA showed an effect of intervention frequency $\left(F_{(2,28)}=40.1, p<0.001\right)$ and time $\left(F_{(5,70)}=17.1, p<\right.$ $0.001)$, but not of group $\left(F_{(1,14)}=0.1, p=0.9\right)$ on biceps brachii CMEP amplitude. There was also an effect of intervention frequency $\times$ time $\left(F_{(4.4,61.1)}=1.3, p<0.001\right)$. Note that CMEP amplitude increased after the TESS and TESS without $5 \mathrm{kHz}$ at 15 $\min (p<0.02), 30 \mathrm{~min}(p<0.001), 45 \mathrm{~min}(p<0.001), 60 \mathrm{~min}$ $(p<0.001)$, and $75 \mathrm{~min}(p<0.01)$ compared with baseline. In contrast, no changes in CMEP amplitude were observed after the sham-TESS session ( $p>0.2$; Fig. $6 A, B$, left column). In addition, repeated-measures ANOVA showed an effect of intervention frequency $\left.\left(F_{(1.2,16.7}\right)=48.8, p<0.001\right)$ and time $\left(F_{(5,70)}=10.7, p<\right.$ $0.001)$ but not group $\left(F_{(1,14)}=0.2, p=0.6\right)$ on biceps brachii MEP amplitude. There was an effect of intervention frequency $X$ time $\left(F_{(3.5,49.6)}=10.9, p<0.001\right)$. Notably, MEP amplitude increased after the TESS without $5 \mathrm{kHz}$ at $15 \min (p<0.001), 30$ $\min (p<0.001), 45 \min (p<0.001), 60 \min (p<0.001)$, and 75 $\min (p<0.001)$ compared with baseline but not after the TESS session ( $p>0.2$ at all time points) and sham-TESS session $(p>$ 0.2 at all time points; Fig. $6 A, B$, middle column). These results together suggest that the $5 \mathrm{kHz}$ carrier frequency had a suppressive effect on corticospinal excitability.

Therefore, we measured the effect of all these conditions on SICI. Repeated-measures ANOVA showed an effect of intervention frequency $\left(F_{(1.4,21.1)}=26.1, p<0.001\right)$ but not time $\left(F_{(5,75)}\right.$ $=2.0, p=0.1)$ or group $\left(F_{(1,15)}=3.0, p=0.1\right)$ on SICI. There was an effect of intervention frequency $\times$ time $\left(F_{(4.9,74.3)}=4.4\right.$, $p<0.001)$. We found that SICI decreased after the TESS at 15 $\min (p<0.001), 30 \mathrm{~min}(p<0.001), 45 \mathrm{~min}(p<0.001), 60 \mathrm{~min}$ $(p<0.001)$, and $75 \mathrm{~min}(p<0.001)$ compared with baseline but not after the TESS without $5 \mathrm{kHz}$ session $(p>0.2$ at all time points) and sham-TESS session ( $p>0.6$ at all time points; Fig. $6 A, B$, right column), supporting the view the $5 \mathrm{kHz}$ carrier frequency had a suppressive effect on cortical activity.

\section{Functional outcomes}

Repeated-measures ANOVA revealed an effect of intervention frequency $\left(F_{(2,18)}=5.5, p<0.05\right)$, task $\left(F_{(2,18)}=8.8, p<0.01\right)$, time $\left(F_{(1,9)}=12.9, p<0.01\right)$, and in their interaction $\left(F_{(4,36)}=\right.$ 7.7, $p<0.001$ ) on the time to complete the task (Fig. $7 A, B$ ). Additionally, there was an effect of intervention frequency $\times$ time $\left(F_{(2,18)}=12.2, p<0.001\right)$ and task $\times$ time $\left(F_{(2,18)}=7.7, p<\right.$ $0.01)$. Our results indicate that the mean time to complete all tasks decreased after TESS $($ coin $=38.1 \pm 5.6 \%, p<0.001$; 
A

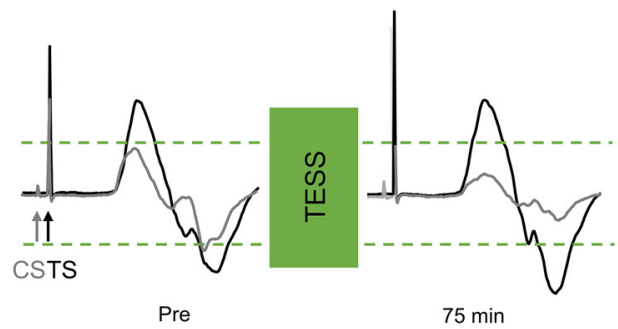

CONTROL
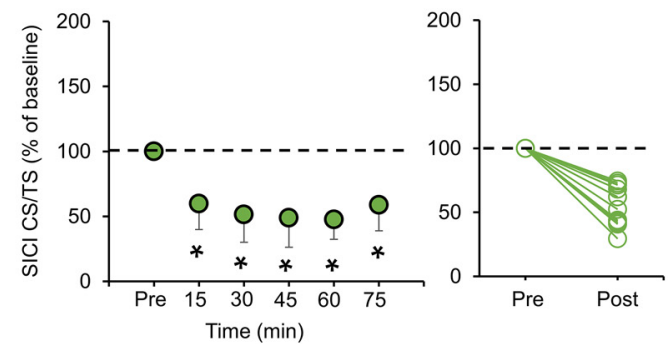

B

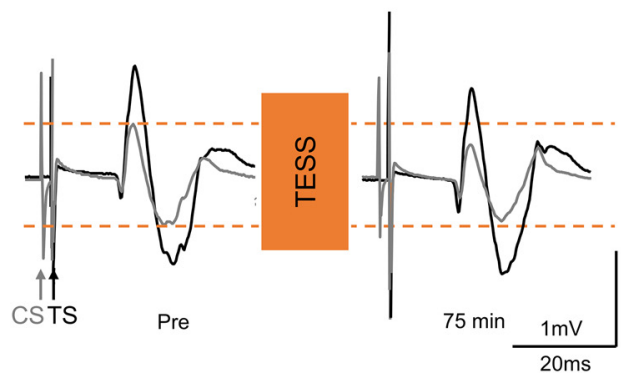

SPINAL CORD INJURY
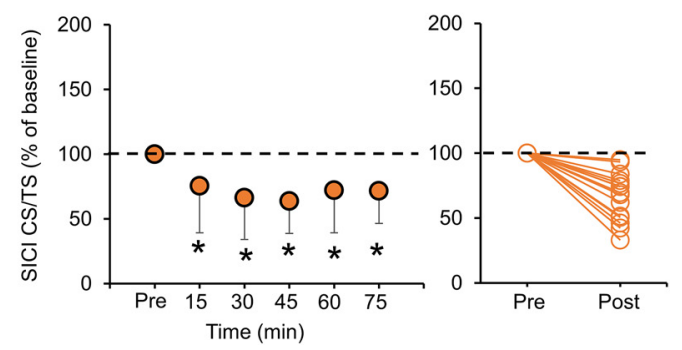

Figure 5. SICI. $\boldsymbol{A}, \boldsymbol{B}$, Averaged traces showing SICI measurements in the biceps brachii muscle in a representative control subject $(\boldsymbol{A})$ and SCI participant $(\boldsymbol{B})$ before and after TESS. Each waveform represents the average of 15 test MEPs (black traces) and 15 conditioned MEPs (gray traces). Arrows at the beginning of each trace indicate the TS and CS used during testing. Graphs show group data (control subjects, $n=15 ; \mathrm{SCl}$ participants, $n=17$ ) and individual data. The ordinate shows the conditioned MEP as a percentage of the test MEP at baseline (percentage of test response) in control subjects and SCI participants before and after TESS (control subjects, green circles; SCI participants, orange circles). The abscissa shows the time of measurements (Pre, and 15, 30, 45, 60, and 75 min after each protocol). In graphs showing data from individual participants, postmeasurements show the average from 15 to 75 min. Error bars indicate SDs. ${ }^{*} p<0.05$.

A

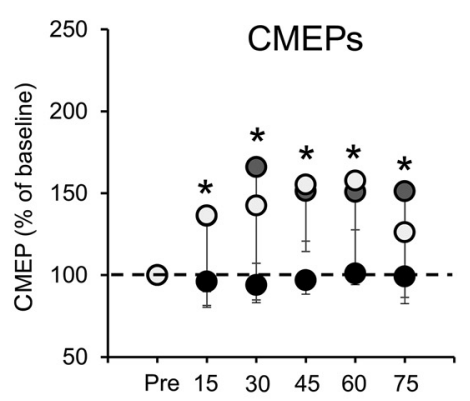

B

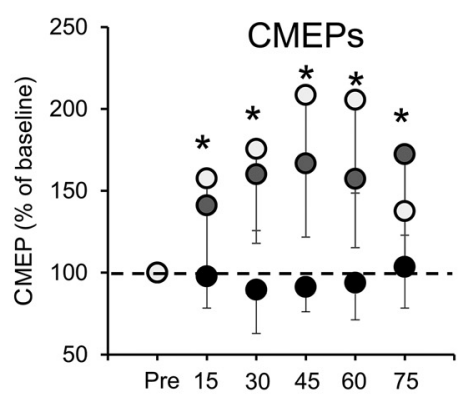

\section{CONTROL}
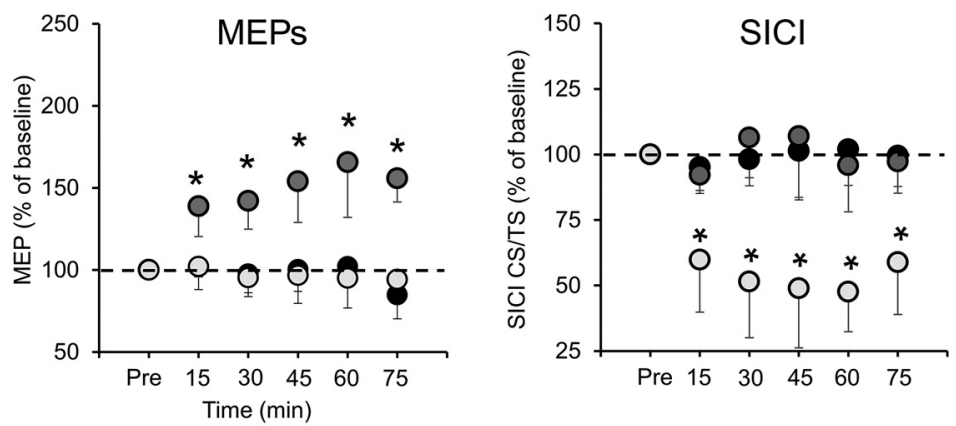

\section{SPINAL CORD INJURY}
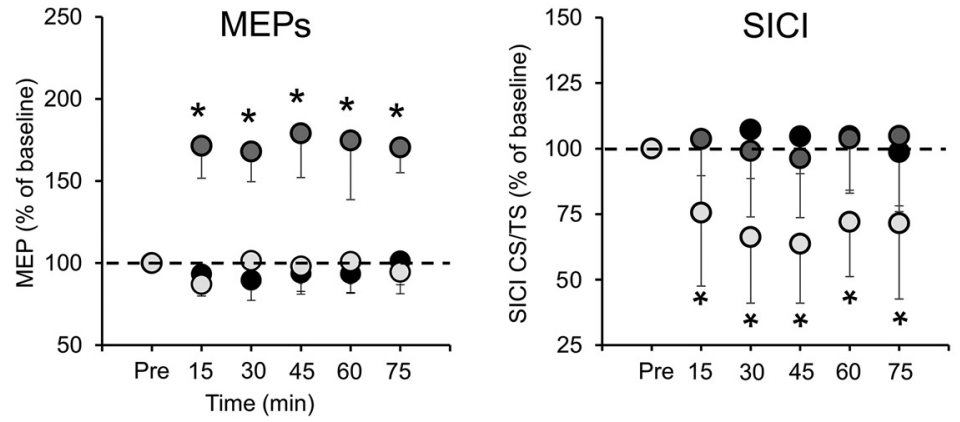

Sham-TESS OTESS w/o 5kHz OTESS

Figure 6. Effect of different currents. $\boldsymbol{A}-\boldsymbol{B}$, Graphs show group data (A. control subjects, $n=8 ; B$. SCI participants, $n=8$ ) for CMEPs MEPs and SICI before and after the TESS, sham-TESS, and TESS applied without the $5 \mathrm{kHz}$ carrier frequency (TESS w/0 $5 \mathrm{kHz}$ ) in control subjects and SCI participants, respectively. The ordinate shows CMEP and MEP amplitude as a percentage of CMEPs and MEPs at baseline (percentage of baseline) and SICl as a percentage of SICl at baseline (percentage of baseline) in control subjects and SCI participants before and after TESS (light gray circles), sham-TESS (black circles), and TESS without $5 \mathrm{kHz}$ (dark gray circles). The abscissa shows the time of measurements (Pre, and 15, 30, 45, 60, and 75 min after each protocol). Error bars indicate SDs. ${ }^{*} p<0.05$. 
A
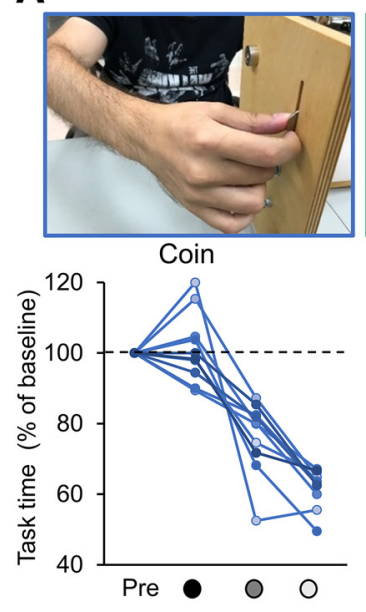
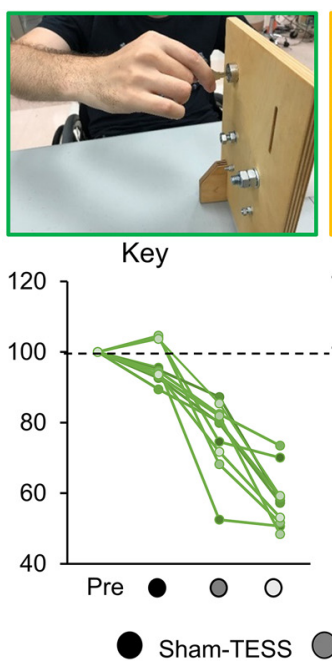

B
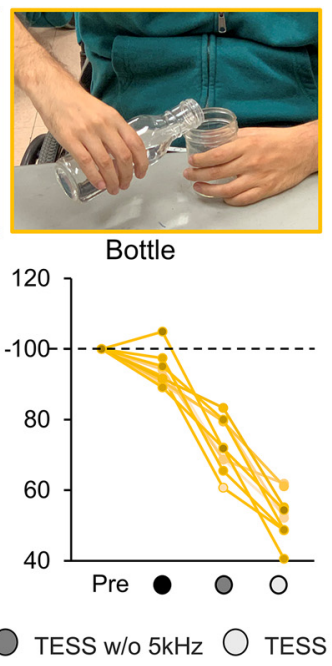

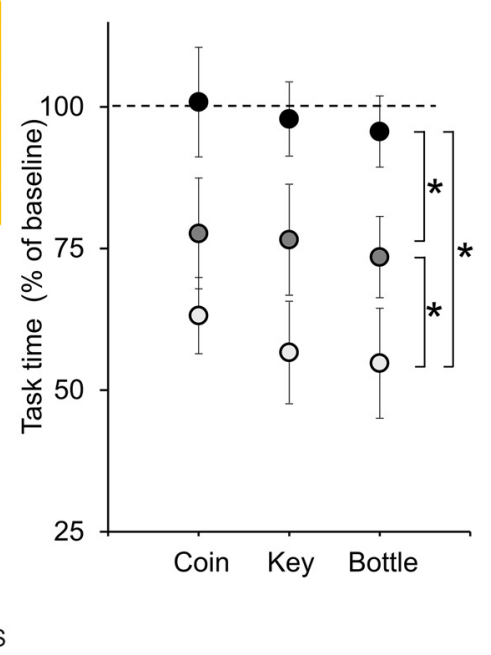

Figure 7. Functional outcomes. $\boldsymbol{A}, \boldsymbol{B}$, Images showing the three subcomponents of the GRASSP (coint, key, and water bottle tests, respectively) tested in SCI participants ( $n=10$ ) before and $\sim 75$ min after sham-TESS, TESS w/0 5 kHz, and TESS. Graphs show individual (left side, A) and group (right side, B). The ordinate shows the time to complete each task as a percentage of the time needed at baseline (percentage of baseline) before and after TESS (light gray circles), sham-TESS (black circles), and TESS without $5 \mathrm{kHz}$ (dark gray circles). The abscissa shows the subcomponents of the GRASSP tested (ccoint, key, and water bottle tests). Error bars indicate SDs. ${ }^{*} p<0.05$.

key $=41.9 \pm 8.2 \%, p<0.001$; and bottle $=47.6 \pm 6.3 \% ; p<$ $0.001)$ and TESS without $5 \mathrm{kHz}($ coin $=22.3 \pm 10.3 \%, p<0.01$; key $=23.6 \pm 10.3 \%, p<0.01$; and bottle $=27.0 \pm 7.4 \%, p<$ $0.001)$ compared with sham-TESS. Notably, the decrease in time was larger after TESS compared with TESS without $5 \mathrm{kHz}$ in the coin $(p<0.01)$, key $(p<0.01)$, and bottle $(p<0.001)$ tasks. In contrast, no differences were observed in the time to complete tasks before and after sham-TESS $(p=0.1)$.

\section{Discussion}

Our novel findings indicate that TESS can change the excitability of cortical and spinal neuronal networks in uninjured and SCI humans. The size of CMEPs but not MEPs increased in proximal and distal arm muscles for 75 min after TESS, but not shamTESS, in control subjects and SCI participants. Paired stimulievoked intracortical inhibition increased after TESS in both groups. These results together suggest that TESS can affect cortical and spinal networks in parallel. When TESS was applied without the $5 \mathrm{kHz}$ carrier frequency, both subcortical and cortical motor-evoked responses were facilitated without changing intracortical inhibition, suggesting that the $5 \mathrm{kHz}$ carrier frequency contributed to the cortical inhibitory effects. Notably, improvements in hand and arm function after TESS was greatest when TESS was used with the $5 \mathrm{kHz}$ carrier frequency. We hypothesized that parallel cortical and subcortical TESS effects can facilitate further recovery of limb function following SCI and suggest that knowledge of the neural basis of TESS-induced recovery may help to maximize its use in rehabilitation.

\section{Cortical and subcortical TESS aftereffects}

Accumulating evidence supports the view that TESS contributes to improve sensory and motor function in humans with SCI (Hofstoetter et al., 2013; Gerasimenko et al., 2015; Gad et al., 2017, 2018; Inanici et al., 2018). Here, for the first time, we examined the effects of TESS applied for several minutes, using parameters currently used in clinical settings, on the excitability of CNS pathways. We found that 20 min of TESS between C5 and C6 spinous processes increased the amplitude of CMEPs but not MEPs elicited by TMS over the primary motor cortex in proximal and distal arm muscles in control subjects and SCI participants. CMEPs likely result from motoneuron activation by descending volleys elicited by excitation of corticospinal axons (Rothwell et al., 1994), reflecting changes in the efficacy of cortico-motoneuronal synapses or motoneuron excitability (Ugawa et al., 1991; Taylor and Gandevia, 2004). CMEP size increases markedly during a voluntary contraction, without changing its latency, which could reflect the excitability of the motoneuron pool linked to monosynaptic connections from corticospinal axons (Ugawa et al., 1995; Taylor et al., 2002). Thus, it is possible that neuromodulation of spinal networks by TESS boosts the excitability of motoneurons and/or cortico-motoneuronal synapses. This is consistent with evidence from electrophysiological (Minassian et al., 2007b; Hofstoetter et al., 2018) and modeling (Rattay et al., 2000; Capogrosso et al., 2013) studies, indicating that a single TESS pulse recruits large-to-medium-diameter proprioceptive and cutaneous afferents within posterior rootlets/roots of different spinal segments. This is also consistent with results from other paradigms showing that 20 min of stimulation targeting the spinal cord is sufficient time to change the excitability of spinal networks (Taylor and Martin, 2009; Bunday and Perez, 2012). The lack of changes in the amplitude of MEPs, while the amplitude of CMEPs increased, was surprising because collision studies using electrical (Ugawa et al., 1991) and magnetic stimulation over the primary motor cortex (Berardelli et al., 1991; Gandevia et al., 1999) indicate that the two stimuli activate similar corticospinal axons. A possibility is that TESS activated inhibitory cortical circuits projecting onto corticospinal neurons. Animal studies showed that afferent input can influence motor cortical activity via dense intracortical projections between the primary motor cortex and the somatosensory cortex (Goldring et al., 1970). Indeed, stimulation of afferent fibers from the somatosensory cortex can produce both excitation and inhibition in motor cortical cells (Porter et al., 1990). To examine the contribution of cortical networks, we assessed SICI by using a randomized paired-pulse TMS paradigm (Kujirai et al., 1993). Studies using pharmacological agents and epidural recordings indicated that SICI reflects the activation of GABA inhibitory circuits in the 
primary motor cortex (Di Lazzaro et al., 2000). We found that SICI increased after TESS in both groups, supporting the view that increased intracortical inhibition could represent a mechanism contributing to the lack of changes in MEP amplitude. In agreement, studies in humans using TMS over the primary motor cortex showed that electrical stimulation of afferent fibers innervating upper limb muscles suppresses corticospinal excitability at interstimulus intervals (ISIs) of $\sim 20-40 \mathrm{~ms}$ (Lei and Perez, 2017) and facilitates corticospinal excitability at ISIs of $\sim 50-100$ ms (Devanne et al., 2009). Afferent inputs reach the primary motor cortex in humans no more than $4 \mathrm{~ms}$ later than the primary somatosensory cortex (Goldring et al., 1970). Thus, even if after SCI conduction velocity in sensory axons is slower (Tan et al., 2007) and the latency of somatosensory-evoked potentials increased (Ozdemir and Perez, 2018), the timing of arrival of afferent information to the primary somatosensory cortex could be sufficient to exert effects on intracortical interneurons projecting to corticospinal neurons controlling upper limb muscles. In addition, all our participants were able to perceive sensory stimuli in dermatomes of the arm and hand tested, indicating the presence of an anatomical substrate to influence cortical inhibitory networks.

In most studies aiming to achieve therapeutic effects in SCI subjects with TESS, a frequency of $5-50 \mathrm{~Hz}$ with a high carrier frequency between 5 and $10 \mathrm{kHz}$ has been applied for several minutes to hours. To further understand the origin of the cortical inhibitory effects induced by TESS, we applied TESS without the $5 \mathrm{kHz}$ carrier frequency. Here, we found that both MEPs and CMEPs were facilitated without changing intracortical inhibition, suggesting that the $5 \mathrm{kHz}$ carrier frequency contributed to the cortical inhibitory effects. This agrees with evidence showing that repeated stimulation of afferent fibers in a peripheral nerve with frequencies between 5 and $50 \mathrm{~Hz}$ usually increases corticospinal excitability (Kaelin-Lang et al., 2002; Golaszewski et al., 2012). Why did the $5 \mathrm{kHz}$ carrier frequency contribute to the cortical inhibitory effects? High carrier frequencies or Russian currents are alternating currents normally within the kilohertz frequency, which are delivered in bursts, with a burst frequency within a physiological range (usually up to $\sim 100 \mathrm{~Hz}$; Ward, 2009). It is thought that this high carrier frequency is beneficial for improving muscle strength (Selkowitz, 1985, 1989) and for suppressing the sensitivity of pain receptors (Ward and Robertson, 1998a,b). High-frequency electrical stimulation of the spinal cord releases serotonin into the dorsal horn (Linderoth et al., 1993), which may depress ascending nociceptive transmission (Fürst, 1999; Millan, 2002). This is also consistent with evidence showing that electrical stimulation of skin afferents at frequencies of $\sim 100 \mathrm{~Hz}$ has inhibitory effects on corticospinal excitability (Mima et al., 2004).

It is interesting that TESS increased inhibition within the primary motor cortex while at the same time improved motor performance in SCI participants. Improved performance after repeated practice and motor learning has been associated with increases in intracortical inhibition in a few cases (Perez et al., 2007). For example, in this study, motor practice involved training participants to improve balanced activation of antagonistic muscles during a cocontraction task. Muscle coactivation ratios between antagonistic muscles of the arm are impaired in humans with SCI compared with control participants (Stahl et al., 2015; Calabro and Perez, 2016). Our observation that TESS applied over C5-C6 spinous processes had similar physiological effects on biceps and triceps brachii muscles suggests that TESS reached neuronal networks controlling antagonistic muscles. This also agrees with evidence showing that electrical stimulation of afferents innervating a flexor muscle could affect intracortical networks contributing to control of the antagonistic extensor muscle (Bertolasi et al., 1998). Thus, changes in muscle coactivation might be a possible factor contributing to our results.

\section{Functional significance}

Our results indicate that a single $20 \mathrm{~min}$ session of TESS at rest is sufficient to change the excitability in neuronal pathways and motor performance as other neuromodulatory paradigms targeting the spinal synapses (Bunday and Perez, 2012) or the primary motor cortex (Roy et al., 2010) in humans with SCI. Previous findings have shown that the effect of TESS on the excitability of neuronal pathways and motor performance are generally similar when measurements are taking before and after the intervention with or without the cervical stimulation after as well as during cervical stimulation in humans (Gad et al., 2018) and rats (Alam et al., 2017). Benefits in motor performance have been reported in humans with SCI when other neuromodulation paradigms, using electrical and/or magnetic stimulation, are used before ( $\mathrm{Lu}$ et al., 2016) or concurrently (Hoffman and Field-Fote, 2007; Potter-Baker et al., 2018) with motor training. Some aspects of the timing of the application of TESS relative to performing the motor task, however, might be important to consider. The responsiveness of the motoneuron pool during voluntary activity also decreases following SCI (Vastano and Perez, 2020), which might benefit from extra inputs recruited by TESS. Our results showing effects on muscles innervated by segments distal to the applied stimulation site suggest that TESS effects extend below targeted segments. This is consistent with previous finding showing that TESS effects spread to adjacent proximal and distal motor pools to the stimulated site after a single pulse (Sayenko et al., 2015). It is likely that the effects of TESS on the interneurons and motor pools projecting to distal hand muscles are the consequence of stimulating afferents from more distal segments, as well as via the high degree of propriospinal intersegmental connectivity and/or to leakage of the current to lower segments due to electrode settings. Thus, a clearer understanding on the neural connectivity underlying the TESS-induced recovery that has been observed may help to maximize rehabilitative strategies.

\section{References}

Alam M, Garcia-Alias G, Jin B, Keyes J, Zhong H, Roy RR, Gerasimenko Y, Lu DC, Edgerton VR (2017) Electrical neuromodulation of the cervical spinal cord facilitates forelimb skilled function recovery in spinal cord injured rats. Exp Neurol 291:141-150.

Awosika OO, Sandrini M, Volochayev R, Thompson RM, Fishman N, Wu T, Floeter MK, Hallett M, Cohen LG (2019) Transcutaneous spinal direct current stimulation improves locomotor learning in healthy humans. Brain Stimul 12:628-634.

Berardelli A, Inghilleri M, Cruccu G, Manfredi M (1991) Corticospinal potentials after electrical and magnetic stimulation in man. Electroencephalogr Clin Neurophysiol Suppl 43:147-154.

Bertolasi L, Priori A, Tinazzi M, Bertasi V, Rothwell JC (1998) Inhibitory action of forearm flexor muscle afferents on corticospinal outputs to antagonist muscles in humans. J Physiol 511:947-956.

Bunday KL, Perez MA (2012) Motor recovery after spinal cord injury enhanced by strengthening corticospinal synaptic transmission. Curr Biol 22:2355-2361.

Calabro FJ, Perez MA (2016) Bilateral reach-to-grasp movement asymmetries after human spinal cord injury. J Neurophysiol 115:157-167.

Capogrosso M, Wenger N, Raspopovic S, Musienko P, Beauparlant J, Bassi Luciani L, Courtine G, Micera S (2013) A computational model for epidural electrical stimulation of spinal sensorimotor circuits. J Neurosci 33:19326-19340.

Devanne H, Degardin A, Tyvaert L, Bocquillon P, Houdayer E, Manceaux A, 
Derambure P, Cassim F (2009) Afferent-induced facilitation of primary motor cortex excitability in the region controlling hand muscles in humans. Eur J Neurosci 30:439-448.

Di Lazzaro V, Oliviero A, Profice P, Pennisi MA, Di Giovanni S, Zito G, Tonali P, Rothwell JC (2000) Muscarinic receptor blockade has differential effects on the excitability of intracortical circuits in the human motor cortex. Exp Brain Res 135:455-461.

Fürst S (1999) Transmitters involved in antinociception in the spinal cord. Brain Res Bull 48:129-141.

Gad P, Gerasimenko Y, Zdunowski S, Turner A, Sayenko D, Lu DC, Edgerton VR (2017) Weight bearing over-ground stepping in an exoskeleton with non-invasive spinal cord neuromodulation after motor complete paraplegia. Front Neurosci 11:333.

Gad P, Lee S, Terrafranca N, Zhong H, Turner A, Gerasimenko Y, Edgerton VR (2018) Non-invasive activation of cervical spinal networks after severe paralysis. J Neurotrauma 35:2145-2158.

Gandevia SC, Petersen N, Butler JE, Taylor JL (1999) Impaired response of human motoneurones to corticospinal stimulation after voluntary exercise. J Physiol 521:749-759.

Gerasimenko Y, Gorodnichev R, Moshonkina T, Sayenko D, Gad P, Reggie Edgerton V (2015) Transcutaneous electrical spinal-cord stimulation in humans. Ann Phys Rehabil Med 58:225-231.

Golaszewski SM, Bergmann J, Christova M, Kunz AB, Kronbichler M, Rafolt D, Gallasch E, Staffen W, Trinka E, Nardone R (2012) Modulation of motor cortex excitability by different levels of whole-hand afferent electrical stimulation. Clin Neurophysiol 123:193-199.

Goldring S, Aras E, Weber PC (1970) Comparative study of sensory input to motor cortex in animals and man. Electroencephalogr Clin Neurophysiol 29:537-550.

Grishin A, Moshonkina T, Solopova I, Gorodnichev R, Gerasimenko Y (2017) A five channel noninvasive electrical stimulator of the spinal cord for rehabilitation of patients with severe motor disorders. Biomed Eng 50:300-304.

Hoffman LR, Field-Fote EC (2007) Cortical reorganization following bimanual training and somatosensory stimulation in cervical spinal cord injury: a case report. Phys Ther 87:208-223.

Hofstoetter US, Hofer C, Kern H, Danner SM, Mayr W, Dimitrijevic MR, Minassian K (2013) Effects of transcutaneous spinal cord stimulation on voluntary locomotor activity in an incomplete spinal cord injured individual. Biomed Tech (Berl) 58:j/bmte.2013.58.issue-s1-A/bmt-20134014/bmt-2013-4014.

Hofstoetter US, McKay WB, Tansey KE, Mayr W, Kern H, Minassian K (2014) Modification of spasticity by transcutaneous spinal cord stimulation in individuals with incomplete spinal cord injury. J Spinal Cord Med 37:202-211.

Hofstoetter US, Freundl B, Binder H, Minassian K (2018) Common neural structures activated by epidural and transcutaneous lumbar spinal cord stimulation: elicitation of posterior root-muscle reflexes. PLoS One 13: e0192013.

Hofstoetter US, Freundl B, Danner SM, Krenn MJ, Mayr W, Binder H, Minassian K (2020) Transcutaneous spinal cord stimulation induces temporary attenuation of spasticity in individuals with spinal cord injury. J Neurotrauma 37:481-493.

Horst M, Heutschi J, van den Brand R, Andersson KE, Gobet R, Sulser T, Courtine G, Eberli D (2013) Multisystem neuroprosthetic training improves bladder function after severe spinal cord injury. J Urol 189:747753.

Inanici F, Samejima S, Gad P, Edgerton VR, Hofstetter CP, Moritz CT (2018) Transcutaneous electrical spinal stimulation promotes long-term recovery of upper extremity function in chronic tetraplegia. IEEE Trans Neural Syst Rehabil Eng 26:1272-1278.

Kaelin-Lang A, Luft AR, Sawaki L, Burstein AH, Sohn YH, Cohen LG (2002) Modulation of human corticomotor excitability by somatosensory input. J Physiol 540:623-633.

Kujirai T, Caramia MD, Rothwell JC, Day BL, Thompson PD, Ferbert A, Wroe S, Asselman P, Marsden CD (1993) Corticocortical inhibition in human motor cortex. J Physiol 471:501-519.

Lei Y, Perez MA (2017) Cortical contributions to sensory gating in the ipsilateral somatosensory cortex during voluntary activity. J Physiol 595: 6203-6217.

Linderoth B, Stiller CO, Gunasekera L, O'Connor WT, Franck J, Gazelius B, Brodin E (1993) Release of neurotransmitters in the CNS by spinal cord stimulation: survey of present state of knowledge and recent experimental studies. Stereotact Funct Neurosurg 61:157-170.

Lu DC, Edgerton VR, Modaber M, AuYong N, Morikawa E, Zdunowski S, Sarino ME, Sarrafzadeh M, Nuwer MR, Roy RR, Gerasimenko Y (2016) Engaging cervical spinal cord networks to reenable volitional control of hand function in tetraplegic patients. Neurorehabil Neural Repair 30: 951-962.

Millan MJ (2002) Descending control of pain. Prog Neurobiol 66:355-474.

Milosevic M, Masugi Y, Sasaki A, Sayenko DG, Nakazawa K (2019) On the reflex mechanisms of cervical transcutaneous spinal cord stimulation in human subjects. J Neurophysiol 121:1672-1679.

Mima T, Oga T, Rothwell J, Satow T, Yamamoto J, Toma K, Fukuyama H, Shibasaki H, Nagamine T (2004) Short-term high-frequency transcutaneous electrical nerve stimulation decreases human motor cortex excitability. Neurosci Lett 355:85-88.

Minassian K, Persy I, Rattay F, Pinter MM, Kern H, Dimitrijevic MR (2007a) Human lumbar cord circuitries can be activated by extrinsic tonic input to generate locomotor-like activity. Hum Mov Sci 26:275-295.

Minassian K, Persy I, Rattay F, Dimitrijevic MR, Hofer C, Kern H (2007b) Posterior root-muscle reflexes elicited by transcutaneous stimulation of the human lumbosacral cord. Muscle Nerve 35:327-336.

Murray LM, Tahayori B, Knikou M (2018) Transspinal direct current stimulation produces persistent plasticity in human motor pathways. Sci Rep 8:717.

Ozdemir RA, Perez MA (2018) Afferent input and sensory function after human spinal cord injury. J Neurophysiol 119:134-144.

Paillard J (1955) Reflexes et regulations d'origine propioceptivechez l'homme. These de Sciences. Paris: Arnette.

Panizza M, Nilsson J, Hallett M (1989) Optimal stimulus duration for the $\mathrm{H}$ reflex. Muscle Nerve 12:576-579.

Perez MA, Lundbye-Jensen J, Nielsen JB (2007) Task-specific depression of the soleus H-reflex after cocontraction training of antagonistic ankle muscles. J Neurophysiol 98:3677-3687.

Phillips AA, Squair JW, Sayenko DG, Edgerton VR, Gerasimenko Y, Krassioukov AV (2018) An autonomic neuroprosthesis: noninvasive electrical spinal cord stimulation restores autonomic cardiovascular function in individuals with spinal cord injury. J Neurotrauma 35:446-451.

Porter LL, Sakamoto T, Asanuma H (1990) Morphological and physiological identification of neurons in the cat motor cortex which receive direct input from the somatic sensory cortex. Exp Brain Res 80:209-212.

Potter-Baker KA, Janini DP, Lin YL, Sankarasubramanian V, Cunningham DA, Varnerin NM, Chabra P, Kilgore KL, Richmond MA, Frost FS, Plow EB (2018) Transcranial direct current stimulation (tDCS) paired with massed practice training to promote adaptive plasticity and motor recovery in chronic incomplete tetraplegia: a pilot study. J Spinal Cord Med 41:503-517.

Radziszewski K (2013) Outcomes of electrical stimulation of the neurogenic bladder: results of a two-year follow-up study. NeuroRehabilitation 32:867-873.

Rattay F, Minassian K, Dimitrijevic MR (2000) Epidural electrical stimulation of posterior structures of the human lumbosacral cord: 2. Quantitative analysis by computer modeling. Spinal Cord 38:473-489.

Rothwell J, Burke D, Hicks R, Stephen J, Woodforth I, Crawford M (1994) Transcranial electrical stimulation of the motor cortex in man: further evidence for the site of activation. J Physiol 481:243-250.

Roy FD, Yang JF, Gorassini MA (2010) Afferent regulation of leg motor cortex excitability after incomplete spinal cord injury. J Neurophysiol 103:2222-2233

Sayenko DG, Atkinson DA, Dy CJ, Gurley KM, Smith VL, Angeli C, Harkema SJ, Edgerton VR, Gerasimenko YP (2015) Spinal segment-specific transcutaneous stimulation differentially shapes activation pattern among motor pools in humans. J Appl Physiol (1985) 118:1364-1374.

Selkowitz DM (1985) Improvement in isometric strength of the quadriceps femoris muscle after training with electrical stimulation. Phys Ther 65: $186-196$

Selkowitz DM (1989) High frequency electrical stimulation in muscle strengthening. A review and discussion. Am J Sports Med 17:103-111.

Stahl VA, Hayes HB, Buetefisch CM, Wolf SL, Trumbower RD (2015) Modulation of hand aperture during reaching in persons with incomplete cervical spinal cord injury. Exp Brain Res 233:871-884. 
Tan AM, Petruska JC, Mendell LM, Levine JM (2007) Sensory afferents regenerated into dorsal columns after spinal cord injury remain in a chronic pathophysiological state. Exp Neurol 206:257-268.

Taylor JL, Gandevia SC (2004) Noninvasive stimulation of the human corticospinal tract. J Appl Physiol (1985) 96:1496-1503.

Taylor JL, Martin PG (2009) Voluntary motor output is altered by spiketiming-dependent changes in the human corticospinal pathway. J Neurosci 29:11708-11716.

Taylor JL, Petersen NT, Butler JE, Gandevia S (2002) Corticospinal transmission after voluntary contractions. Adv Exp Med Biol 508: 435-441.

Ugawa Y, Rothwell JC, Day BL, Thompson PD, Marsden CD (1991) Percutaneous electrical stimulation of corticospinal pathways at the level of the pyramidal decussation in humans. Ann Neurol 29:418-427.

Ugawa Y, Terao Y, Hanajima R, Sakai K, Kanazawa I (1995) Facilitatory effect of tonic voluntary contraction on responses to motor cortex stimulation. Electroencephalogr Clin Neurophysiol 97:451-454.

Vastano R, Perez MA (2020) Changes in motoneuron excitability during voluntary muscle activity in humans with spinal cord injury. J Neurophysiol 123:454-461.

Ward AR (2009) Electrical stimulation using kilohertz-frequency alternating current. Phys Ther 89:181-190.

Ward AR, Robertson VJ (1998a) Sensory, motor, and pain thresholds for stimulation with medium frequency alternating current. Arch Phys Med Rehabil 79:273-278.

Ward AR, Robertson VJ (1998b) Variation in torque production with frequency using medium frequency alternating current. Arch Phys Med Rehabil 79:1399-1404.

Ward NJ, Kassal I, Aspuru-Guzik A (2009) Preparation of many-body states for quantum simulation. J Chem Phys 130:194105. 